\title{
Femtosecond laser-assisted removal of an intracorneal chestnut, a case report
}

Yong Jie Qin ${ }^{1}$, Jin Zeng ${ }^{1}$, Hong Liang Lin ${ }^{1}$, Wen Juan Xie ${ }^{1}$, Yan Zhang ${ }^{1}$, Hai Ke Guo ${ }^{2^{*}}$ and Hong Yang Zhang ${ }^{1^{*}}$

\begin{abstract}
Background: To report a case of femtosecond laser-assisted removal of an intracorneal chestnut.

Case presentation: A chestnut was obliquely protruding to the stroma of cornea and it was localized at the paracentral region on the left eye of a 32-year-old man. The best-corrected visual acuity (BCVA, in decimal values) was 0.6 in the injured eye. The white ulcers with feathery edges or satellite infiltrates were not observed in the lesion, and the anterior chamber was deep and quiet. Anterior segment optical coherence tomography (AS-OCT) demonstrated that the original entry path of the foreign body had been sealed, spanning a thickness of approximate $152 \mu \mathrm{m}$. In view of location of the intraocular chestnut at the paracentral region, femtosecond laser was applied according to the procedures of IntraLase Enabled Keratoplasty (IEK) to create an anterior lamellar flap rapidly and precisely. The lamellar flap was easily separated with a flap lifter, and the chestnut was removed entirely using a pair of forceps. In 3 days after surgery, the patient complained of mild pain and blurred vision. These symptoms were relieved after treatment with the eyedrops. At three-month follow-up, the corneal wound was healed well, and the BCVA was greatly improved to 1.2 in the left eye. A dot-like haze was observed corresponding to the scar at the site of foreign body removal. No surgical induction of corneal astigmatism was found in the corneal topography.

Conclusions: Without induction of a visually significant scar and corneal astigmatism, the IEK procedure of femtosecond laser is of particular interest as it provides a unique method for removal of intracorneal foreign bodies impinging on the visual axis.
\end{abstract}

Keywords: Femtosecond laser, IEK, Corneal injury, Intracorneal foreign body, Chestnut

\section{Background}

Corneal foreign bodies are a common type of eye injury involving the foreign substances, such as metals, glasses or organic materials that superficially adhere to or embed in the cornea. The well tolerated substances without precipitating a pathological reaction to cornea can be left in the cornea and followed up regularly [1]. Some objects that are poorly tolerated by the patients must be promptly removed. Surgeries are suggested to remove the substance that is localized at the paracentral region and causing vision loss [2]. However, aggressive attempts to remove deep fragments may cause a visually significant scar and result in undesirable distortion of

\footnotetext{
* Correspondence: guohaike@hotmail.com; hy3005716@163.com

${ }^{2}$ Shanghai Heping Eye Hospital, 61 Shanghai Yiminhe Road, Shanghai, China 'Department of Ophthalmology, Guangdong Eye Institute, Guangdong General Hospital and Guangdong Academy of Medical Sciences, 106 Zhongshan Er Road, Guangzhou 510080, China
}

the corneal topography [3]. Femtosecond laser is an accurate technology to make extremely smooth and precise cuts. It has been commonly used in corneal refractive surgery [4]. Here we performed a reliable procedure using femtosecond laser to remove an intracorneal chestnut.

\section{Case presentation}

A 32-year-old man presented with the symptoms of foreign body sensation and blurred vision in the left eye 3 days before presentation. The best-corrected visual acuity (BCVA, in decimal values) was 1.2 in the right eye and 0.6 in the left eye. Slit-lamp biomicroscopy (BX-900, Haag-Streit AG, Koeniz, Switzerland) of the left eye revealed an intracorneal foreign body, localized at the paracentral region, obliquely protruding to Descemet membrane with no penetration into the anterior chamber (Fig. 1). The original entry path of the foreign body

(c) The Author(s). 2018 Open Access This article is distributed under the terms of the Creative Commons Attribution 4.0 International License (http://creativecommons.org/licenses/by/4.0/), which permits unrestricted use, distribution, and 




Fig. 1 a Slit-lamp examination demonstrated a chestnut embedded in the corneal stroma (arrow). b The chestnut was obliquely protruding to Descemet membrane but not penetrating to the anterior chamber (arrow). c The white line through the chestnut (arrowhead) indicated the position of AS-OCT imaging probe. The shadow (asterisk) shown in AS-OCT corresponded to the location of the chestnut. Analysis with caliper tool showed an approximate $152 \mu \mathrm{m}$ thickness of sealed tissue at the original entry path of the chestnut. AC,

anterior chamber

had sealed and epithelialized, leaving a sub-epithelial opacity and edematous stroma (Fig. $1 \mathrm{~b}$ and c). The shadow effect shown in the anterior segment optical coherence tomography (AS-OCT, RTVue XR, Optovue, Inc., Fremont, $\mathrm{CA}$ ) corresponded to the location of the intracorneal chestnut (Fig. 1c). The corneal thickness was approximate $755 \mu \mathrm{m}$ at the site of lesion, of which $152 \mu \mathrm{m}$ distance from the sealed corneal epithelium to the chestnut (Fig. 1c). The white ulcers with feathery edges or satellite infiltrates were not observed. The intraocular pressure, anterior chamber, lenses and the fundi appeared normal. No signs of systemic disorders were found in the presented case.

This study conformed to the principles of the Declaration of Helsinki and was approved by the Institutional Ethics Committee of Guangdong General Hospital and Guangdong Academy of Medical Sciences. After discussing with the patient and informed consent was obtained, femtosecond laser was applied using the protocols of IntraLase Enabled Keratoplasty (IEK, $\mathrm{iFS}^{\mathrm{m}}$ Advanced Femtosecond Laser System). Following parameters were used: $300 \mu \mathrm{m}$ lamellar depth, $7.5 \mathrm{~mm}$ diameter, $1.20 \mu \mathrm{J}$ energy, and cut angle with 180 degrees from 12 o'clock to 6 o'clock in the left eye (Fig. 2a). Under strict aseptic precautions, IEK was performed to create an anterior lamellar flap according to the routine procedures. The lamellar flap was easily separated with a flap lifter to expose the superior side of the chestnut. As shown in
Fig. 2a, the chestnut was then removed entirely with a pair of forceps under a surgical microscope (OPMI LUMERA 700, Carl Zeiss Meditec, Jena, Germany). After removal of the chestnut, no fluorescein leakage was found with the Seidel test and the wound was washed with $250 \mathrm{~mL}$ normal saline containing $40 \mathrm{mg}$ gentamycin. The edges were dried for 3 min using a surgical sponge, and a soft contact lens (Extended wear, PureVision. Bausch and Lomb, NY) was applied over the surface. No suture was applied. The chestnut, shown in Fig. 2b, was inoculated onto Sabouraud glucose agar and chocolate agar to detect potential growth of fungi and bacteria.

Before the surgery, Levofloxacin (Santen Pharmaceutical Co., Ltd. Japan) was prescribed for four times per day, and postoperatively, TobraDex (tobramycin 0.3\% and dexamethasone $0.1 \%$, s.a. Alcon-Couvreur n.v. Puurs, Belgium) was included and tapered weekly over a month. In 3 days after surgery, the patient complained of mild pain and blurred vision. These symptoms were relieved after treatment with the eyedrops. At three-month follow-up, a dot-like haze was noted in the cornea, which was corresponded to the scarring formation at the site of foreign body removal (Fig. 2c and d). As shown in Fig. 3, assessment with corneal topography (Oculus Pentacam Typ 70,700, Topcon, Tokyo, Japan) demonstrated that there was no surgical induction of corneal astigmatism compared to the preoperative 


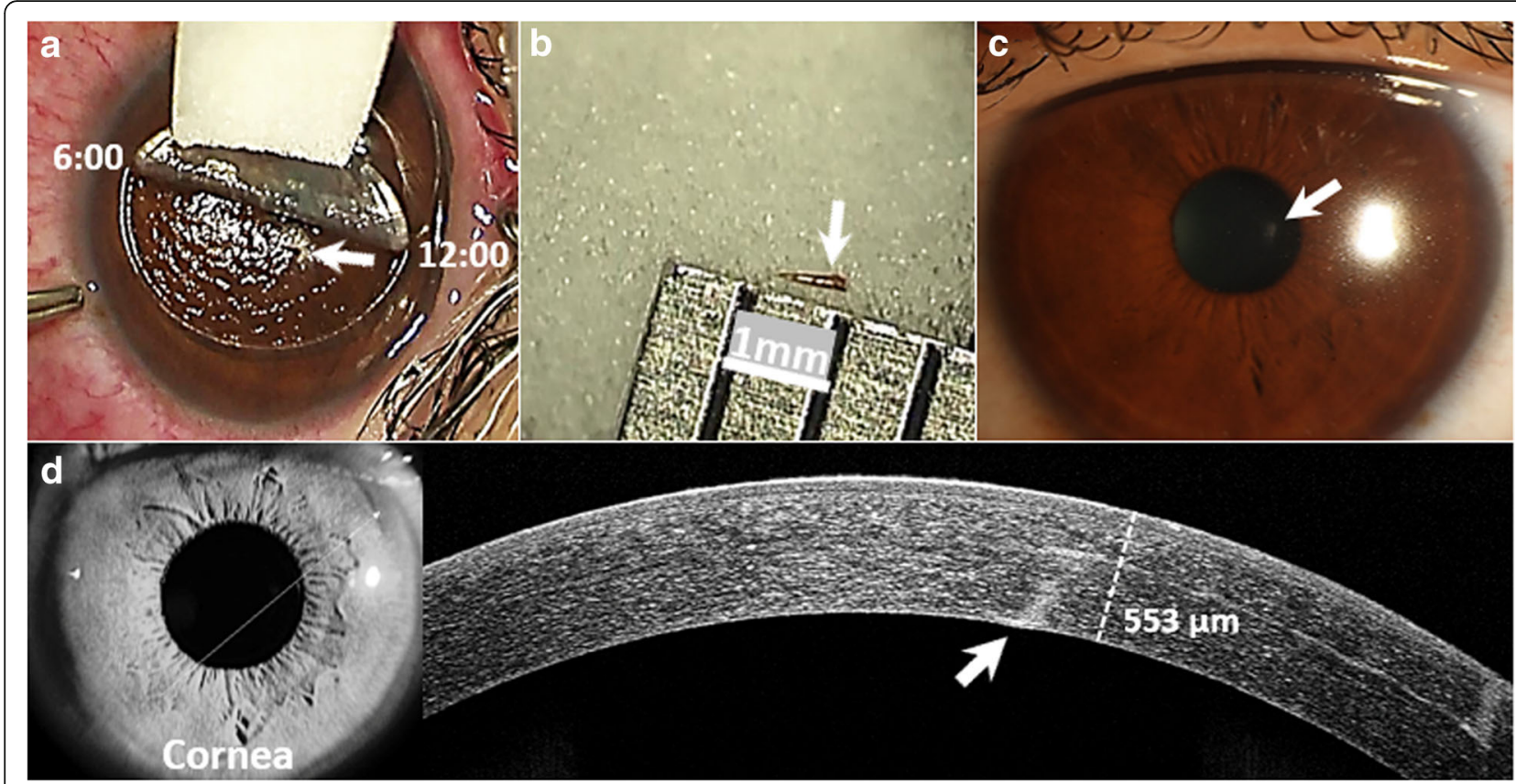

Fig. 2 a Anterior lamellar flap of the cornea was created from 12:00 to 6:00 in the left eye to expose the chestnut (arrow). b A chestnut, around $600 \mathrm{~mm}$ in diameter, was removed completely (arrow). c Three months after surgery, a dot-like haze was noted at the site of the chestnut removal (arrow). $\mathbf{d}$ The white line indicated the position of AS-OCT assessment. A hyper-reflectivity illustrated in AS-OCT was corresponded to the haze that caused by the chestnut (arrow). The corneal thickness was $553 \mu \mathrm{m}$ at three-month follow-up

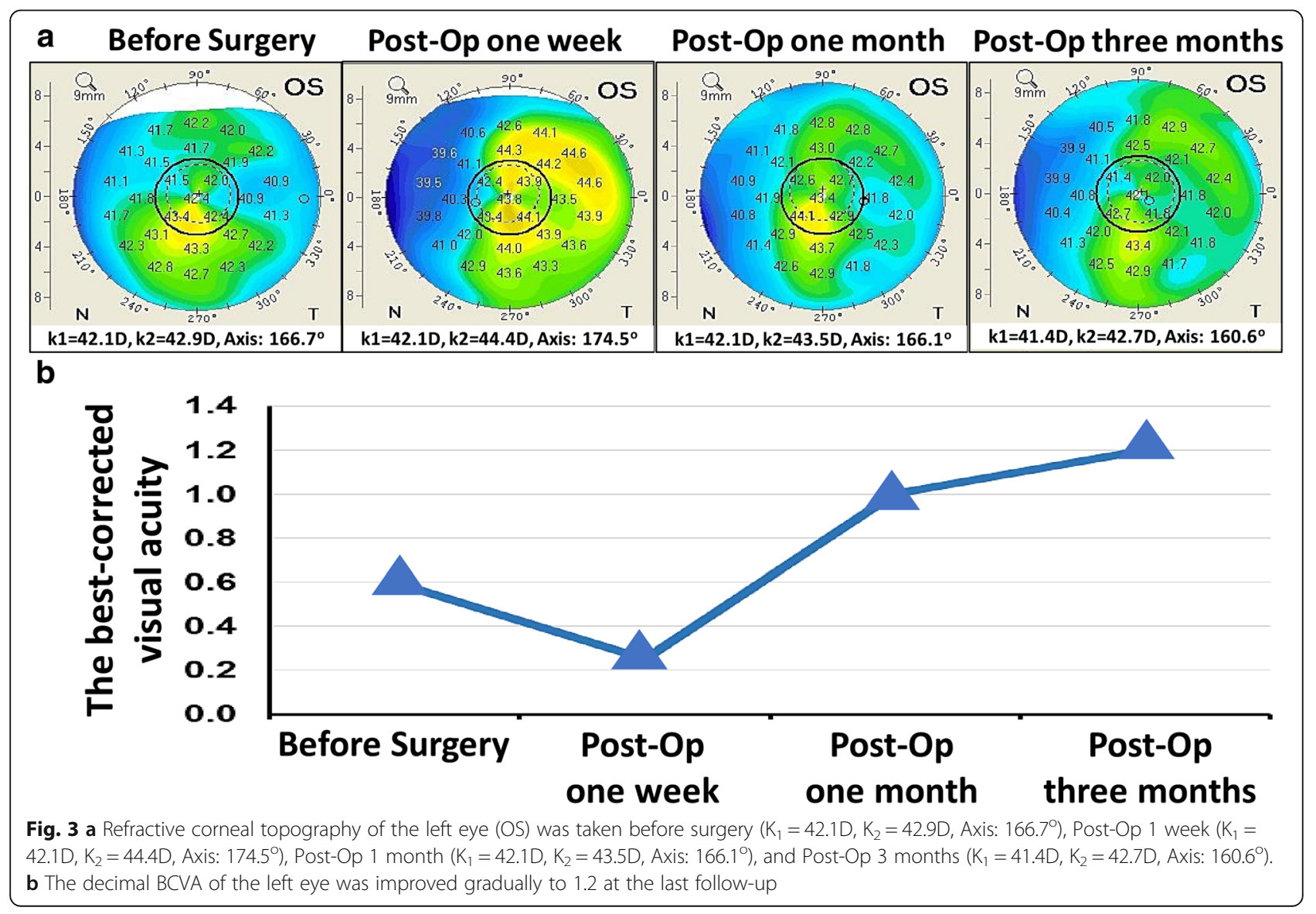


astigmatism (Pre-Op, $K_{1}=42.1 \mathrm{D}, \mathrm{K}_{2}=42.9 \mathrm{D}$, Axis: $166.7^{\circ}$ vs 3 -month Post-Op, $K_{1}=41.4 \mathrm{D}, \mathrm{K}_{2}=42.7 \mathrm{D}$, Axis: $160.6^{\circ}$ ), and the Post-Op decimal BCVA in the left eye was improved gradually from 0.3 to 1.2 . The results in microbiological culture of fungi and bacteria were negative.

\section{Discussion and conclusion}

Conventionally, removal of the intracorneal foreign bodies was manipulated with a scalpel blade and a needle. This technique required closing the peripheral lamellar pocket with suture, which may induce a visually significant scar [5]. A concern in this case was that the BCVA of the injured eye was still good enough and the chestnut embedded in the stroma was localized at the paracentral region. Inappropriate manipulations to dislodge it could induce visual impairment. A simple and effective technique was introduced to remove the corneal foreign body with a suture needle [6]. But it was considered unsuitable in our case because the original entry path of the chestnut had been sealed. Femtosecond laser generated sutureless anterior lamellar incisions, and improved the vision with rapid visual rehabilitation and no significant astigmatism induction [4, 7]. It was recently reported to remove a corneal scar caused by an impacted stromal glass [8]. Therefore, femtosecond laser was adopted in our case to create an anterior corneal flap. The desired depth and cut angle allowed us to remove the chestnut effectively. Of note, his BCVA was greatly improved, and the large and deep lamellar incision did not change the refractive error and did not induce scaring formation. Moreover, the chestnut was removed entirely, and no fluorescein leakage was observed in the wound, supporting that the procedure was extremely safe without disrupting the chestnut and causing penetration of the foreign body in ocular tissues.

The AS-OCT imaging demonstrated that the entry wound of the chestnut had been sealed with approximate $152 \mu \mathrm{m}$ thickness, indicating that the lamellar-cut depth should be greater than that in order to grasp the chestnut. We thereby created an anterior lamellar flap with $300 \mu \mathrm{m}$ depth to expose the free superior edge of the chestnut. Our results demonstrated that the chestnut can be simply removed with a pair of forceps. Thus, AS-OCT is a valuable tool for management of a corneal foreign body.

The concern with the use of femtosecond laser to remove the chestnut was the spreading of infections. The mature chestnut contains clusters of sharp and yellow-brown thorns that might harbor filamentous fungi leading to fungal keratitis [9]. In this case, the fungal infection was excluded. Because no sign of infections was noted, the cornea and the anterior chamber seemed to be quiet after injury, and postoperatively, the corneal wound was healed well, and no causative agent was found in the microbiological investigation. However, a careful examination is suggested to exclude fungal and bacterial infection before the surgery.

In conclusion, the femtosecond laser-assisted IEK is a precise procedure to create a lamellar flap without distorting the corneal topography. Our results suggest that femtosecond femtolaser-assisted IEK is a promising technique for the removal of intracorneal foreign bodies, particularly for those embedded in the paracentral region.

\section{Abbreviations}

AC: Anterior chamber; AS-OCT: Anterior segment optical coherence tomography; BCVA: Best-corrected visual acuity; IEK: IntraLase Enabled Keratoplasty; Post-Op: Post-operative

\section{Acknowledgements}

The authors thank the patient who generously agreed to participate in this medical report.

\section{Funding}

National Natural Science Foundation of China (Grant No. 81600752 and 81400416); Science and Technology Program of Guangzhou (Grant No. 201607010390)

Availability of data and materials

All data generated or analysed during this study are included in this published article.

\section{Authors' contributions}

$Y J Q, H L L$, and WJX performed the imaging of the patient and acquired the data. YJQ, ZJ, YZ, and HYZ designed the procedures of IntraLase Enabled Keratoplasty to remove the chestnut and followed up with the patient. YJQ, HKG, and HYZ wrote the manuscript and analyzed the data. HKG and $\mathrm{HYZ}$ assisted in revising manuscript and also offered critical advice regarding discussion. All authors read and approved the final manuscript.

\section{Ethics approval and consent to participate}

This study has been performed in accordance with the Declaration of Helsinki and was approved by the Research Ethics Committee of Guangdong General Hospital and Guangdong Academy of Medical Sciences.

\section{Consent for publication}

Written informed consent was obtained from the patient for publication of the clinical data was obtained from the patient. A copy of the written consent is available for review by the editor of this journal.

\section{Competing interests}

The authors declare that they have no competing interests.

\section{Publisher's Note}

Springer Nature remains neutral with regard to jurisdictional claims in published maps and institutional affiliations.

Received: 26 April 2018 Accepted: 3 August 2018

Published online: 28 August 2018

\section{References}

1. Covert DJ, Henry CR, Sheth BP. Well-tolerated intracorneal wood foreign body of 40-year duration. Cornea. 2009;28:597-8.

2. Celebi AR, Kilavuzoglu AE, Altiparmak UE, et al. The role of anterior segment optical coherence tomography in the management of an intra-corneal foreign body. Springerplus. 2016;5:1559.

3. Chen WL, Tseng CH, Wang IJ, et al. Removal of semitranslucent cactus spines embedded in deep cornea with the aid of a fiberoptic illuminator. Am J Ophthalmol. 2002;134:769-71. 
4. Kymionis GD, Kankariya VP, Plaka AD, et al. Femtosecond laser technology in corneal refractive surgery: a review. J Refract Surg. 2012;28:912-20.

5. Au YK, Libby C, Patel JS. Removal of a corneal foreign body through a lamellar corneal pocket. Ophthalmic Surg Lasers. 1996;27:471-2.

6. Yang X. Removal of corneal foreign bodies that project into the anterior chamber: use of a suture needle. Am J Ophthalmol. 2000;129:801-2.

7. Shousha MA, Yoo SH, Kymionis GD, et al. Long-term results of femtosecond laser-assisted sutureless anterior lamellar keratoplasty. Ophthalmology. 2011; 118:315-23.

8. Taneja M, Rathi VM, Bagga B. One-stage femtolaser-assisted lamellar keratoplasty and foreign body removal. Optom Vis Sci. 2015;92:e42-5.

9. Cho KJ, Kim MS. Fungal hyphae growing into anterior chamber from cornea. Can J Ophthalmol. 2014:49:e151-4.

Ready to submit your research? Choose BMC and benefit from:

- fast, convenient online submission

- thorough peer review by experienced researchers in your field

- rapid publication on acceptance

- support for research data, including large and complex data types

- gold Open Access which fosters wider collaboration and increased citations

- maximum visibility for your research: over $100 \mathrm{M}$ website views per year

At BMC, research is always in progress.

Learn more biomedcentral.com/submissions 